\title{
Paratesticular dedifferentiated liposarcoma with leiomyosarcomatous differentiation: a case report with a review of literature
}

\author{
Kazuhito Hatanaka, Takako Yoshioka, Takashi Tasaki and Akihide Tanimoto*
}

\begin{abstract}
Paratesticular liposarcoma is a rare neoplasm, described in single case studies or components of larger studies, as histologically well-differentiated liposarcoma (WDL) and dedifferentiated liposarcoma (DL). However, leiomyosarcomatous differentiation is an extremely rare occurrence in WDL and DL. We report a case of leiomyosarcomatous differentiation in a 77-year-old man. The patient presented with a painless right scrotal mass, Magnetic resonance imaging showed a large mass along the right spermatic cord. The resected mass, measuring $17.5 \times 12 \times 5 \mathrm{~cm}$, was composed of a high-grade pleomorphic undifferentiated sarcomatous component with necrosis. Atypical smooth muscle differentiation was also detected. Additional tumor sampling revealed the presence of a WDL component. Immunohistochemical analysis of the pleomorphic sarcomatous component showed positive staining for MDM2 and CDK4, and negative staining for alpha smooth muscle actin (aSMA) and desmin. The smooth muscle component was positive for aSMA and desmin, and negative for MDM2 and CDK4. Extension from primary retroperitoneal sarcoma was not proved. We diagnosed of $D L$ with leiomyosarcomatous differentiation.
\end{abstract}

Virtual slides: The virtual slide(s) for this article can be found here: http://www.diagnosticpathology.diagnomx.eu/ vs/1484291498104021.

Keywords: Dedifferentiated liposarcoma, Leiomyosarcomatous differentiation, Paratesticular region

\section{Background}

Liposarcoma is the most common type of soft-tissue sarcoma, accounting for about $20 \%$ of all mesenchymal malignant tumors. It usually arises from the thigh and retroperitoneum. However, pleomorphic liposarcomas arising in the foot and ankle have been reported [1]. The occurrence of liposarcomas in the paratesticular regions is also rare [2]. A study of 30 cases of paratesticular liposarcoma showed that 19 cases were well-differentiated liposarcoma (WDL), 10 cases were dedifferentiated liposarcoma (DL), and 1 showed a myxoid/round cell liposarcoma [2]. DL is usually composed of atypical lipomatous tumor (ALT)/WDL areas and dedifferentiated components that usually overlap with spindle/pleomorphic cell high-grade sarcoma or myxoid/spindle cell low-grade sarcoma. Dedif-

\footnotetext{
*Correspondence: akit09@m3.kufm.kagoshima-u.ac.jp Department of Molecular and Cellular Pathology, Kagoshima University Graduate School of Medical and Dental Sciences, 8-35-1 Sakuragaoka, Kagoshima 890-8544, Japan
}

(c) 2013 Hatanaka et al.; licensee BioMed Central Ltd. This is an Open Access article distributed under the terms of the Creative Commons Attribution License (http://creativecommons.org/licenses/by/2.0), which permits unrestricted use, distribution, and reproduction in any medium, provided the original work is properly cited. ferentiated areas rarely show heterologous differentiation with myogenic, osteo/chondrosarcomatous, or angiosarcomatous elements [3]. Moreover, the peculiar meningotheliallike whorling and metaplastic bone formation were reported as other elements of heterologous differentiation [4]. Leiomyosarcoma, rhabdomyosarcoma, chondrosarcoma, and osteosarcoma differentiation has also been reported in malignant mesenchymal tumor [5].

Paratesticular liposarcoma with leiomyosarcomatous differentiation is extremely rare; to our knowledge, only single case studies or components of larger studies have been reported in the English literature [6-9]. Here, we report a very rare case of paratesticular DL with leiomyosarcomatous differentiation with a review of literature.

\section{Case presentation}

A 77-year-old man with diabetes mellitus presented with a growing mass in the right testis of 3 months duration. A computed tomographic scan revealed a soft tissue 
mass, measuring $12 \mathrm{~cm}$ in diameter, in the right scrotal sac. The mass showed low signal intensities on T2-weighted magnetic resonance imaging. Radical inguinal orchiectomy was performed because of suspected malignancy.

\section{Materials and methods}

The resected specimens were fixed with 10\% neutralbuffered formalin and embedded in paraffin blocks. Four micrometer-thick paraffin sections were cut and mounted on glass slides, deparaffinized in xylene, rehydrated with ethanol, and immunostained with the following antibodies: MDM2 (monoclonal, 1B10, Novocastra, Newcastle upon Tyne, UK), CDK4 (monoclonal, DCS-31, Invitrogen, Camarillo, CA), S-100 protein (polyclonal, Z0311, Dako, Glostrup, Denmark), alpha smooth muscle actin ( $\alpha \mathrm{SMA})$ (monoclonal, $\alpha \mathrm{sm}-1$, Novocastra), desmin (monoclonal, D33, Dako, Glostrup, Denmark), and MyoD1 (monoclonal, 5.8A, Dako, Glostrup, Denmark). Sections were stained using Dako Envision kit (Dako, Glostrup, Denmark).

\section{Results}

On macroscopic examination, a nodular mass measured $17.5 \times 12 \times 5 \mathrm{~cm}$, and showed a white-yellow to redbrown cut surface with marked necrosis (Figure 1). The distribution of adipose tissue was unclear in the tumor. Microscopic examination revealed that the tumor was composed mainly of proliferating atypical short spindle or oval cells that resembled high-grade undifferentiated sarcoma (Figure 2A). The cells showed relatively high mitotic activity with more than 5 mitoses per 10 highpower fields. Necrosis and hemorrhage were also present, and necrosis was observed in approximately $40 \%$ of the

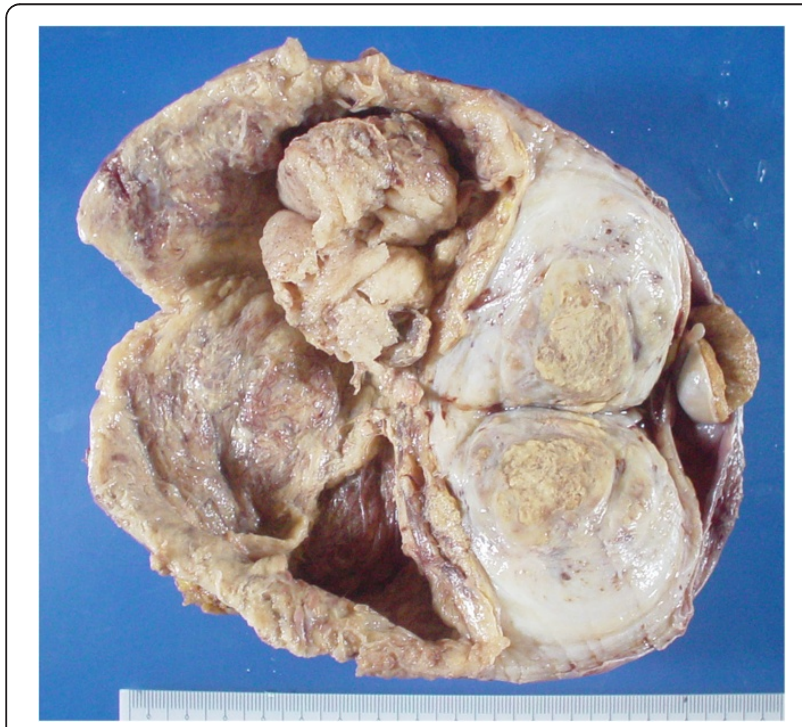

Figure 1 Macroscopic tumor characteristics. Nodular mass showing a white-yellow to red-brown cut surfaces with marked necrosis.

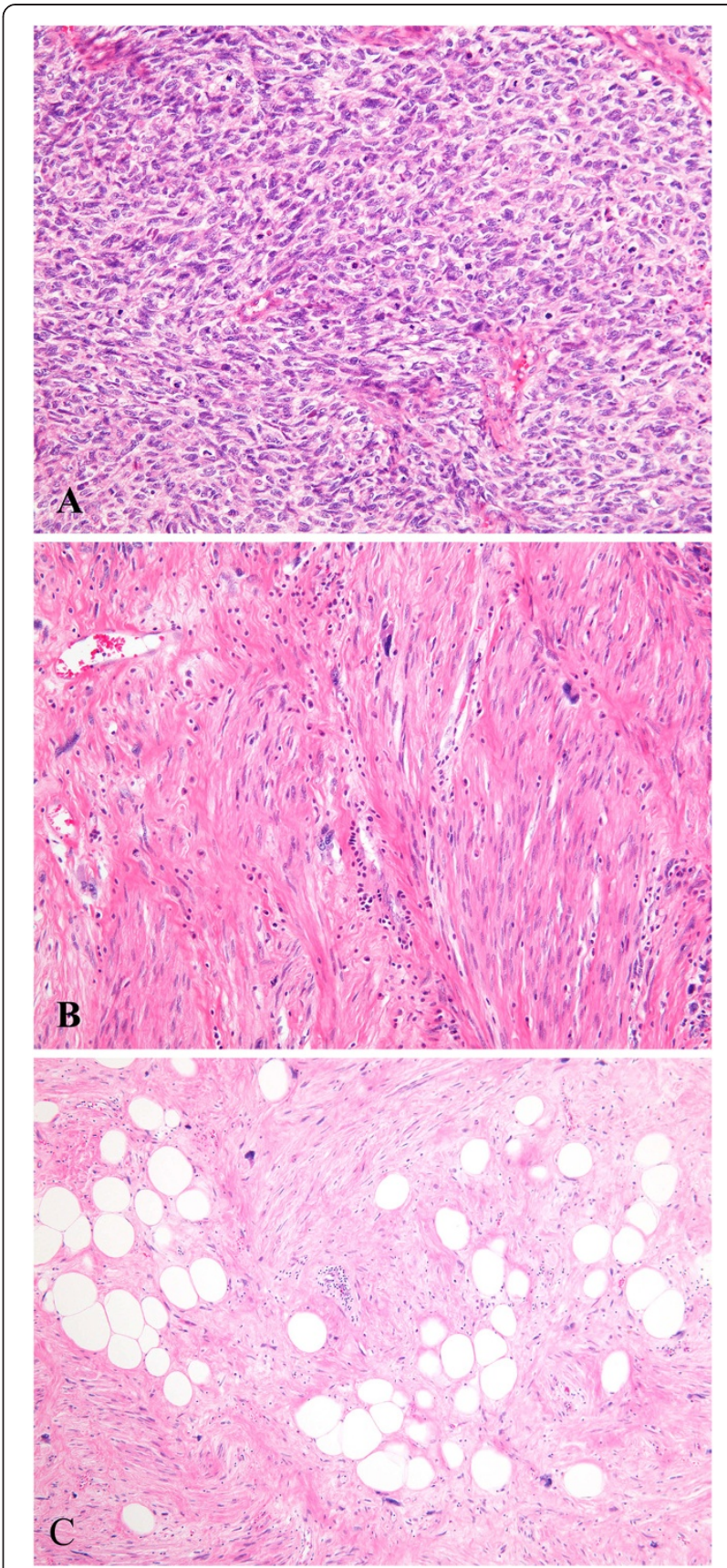

Figure 2 Microscopic tumor characteristics. (A) Proliferation of atypical short spindle or oval cells resembling high grade undifferentiated sarcoma. (B) Proliferating spindle cells containing elongated, blunt-ended nuclei and eosinophilic fibrillar cytoplasm with scattered enlarged and irregular nuclei. (C) The presence of a WDL component, showing mature-appearing adipose tissue and fibrous bands with irregular nuclei.

tumor. Moreover, fascicles of spindle cells containing elongated, blunt-ended nuclei and eosinophilic fibrillar cytoplasm with scattered enlarged and irregular nuclei were also detected (Figure 2B). Osteo/chondrosarcomatous differentiation and meningothelial-like whoring pattern were 


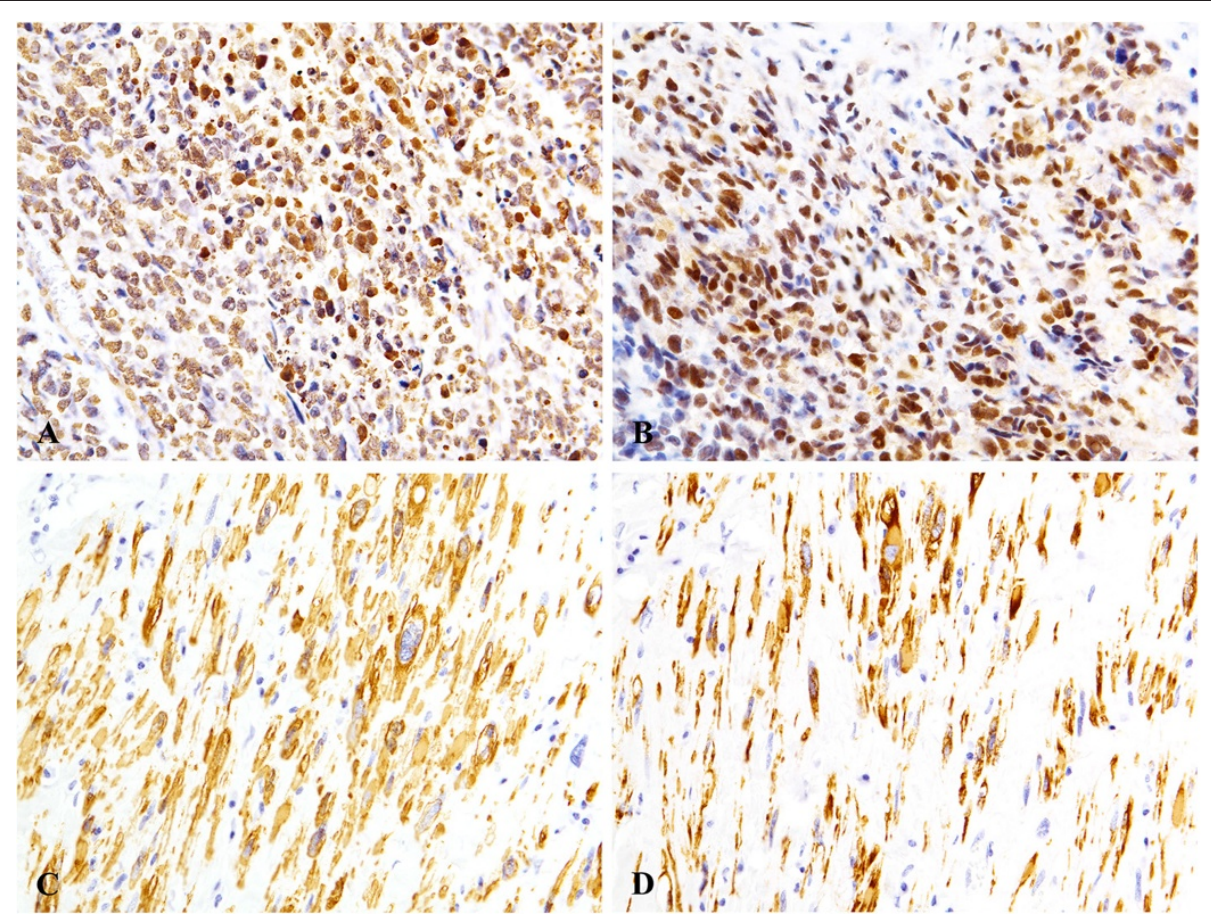

Figure 3 Immunohistochemical analysis. (A)(B) Sarcomatous component is positive for MDM2 (A) and CDK4 (B). (C)(D) Spindle cells are positive for alpha smooth muscle actin (C) and desmin (D)

not observed. The presence of the WDL component was proved in additional samples from the tumor (Figure 2C).

On immunohistochemical staining, the high-grade sarcomatous component was positive for MDM2 (Figure 3A) and CDK4 (Figure 3B), but not for S-100 protein, $\alpha$ SMA, and desmin. The spindle cell component was positive for $\alpha$ SMA (Figure 3C) and desmin (Figure 3D), but not for MDM2 and CDK4. Both components showed no reactivity to MyoD1. The resected margin was free, and extension from primary retroperitoneal sarcomas was not proved.

DL with leiomyosarcomatous differentiation was diagnosed. The leiomyosarcomatous component occupied approximately $10 \%$ of the tumor. The patient was disease free after at the 2-year follow-up.

\section{Discussion}

Paratesticular liposarcoma is rare and has been reported in single case reports or as components of larger series.
These tumors are thought to arise de novo from the adipose tissue around the spermatic cord or by malignant transformation of pre-existing lipomas [10]. In 2003, Montogomery et al studied 30 cases of paratesticular liposarcomas in men aged $41-87$ years (mean age, 63 years) [2]. The tumors involved the spermatic cord (23 cases), testicular tunics ( 6 cases), or epididymis ( 1 case) and measured from $3-30 \mathrm{~cm}$ (mean, $11.7 \mathrm{~cm}$ ). From the histological viewpoint, 19 cases were WDL, 10 were DL, and 1 was a myxoid/round cell liposarcoma. Among $10 \mathrm{DL}$ cases, 5 were high-grade and 5 were low-grade. Leiomyosarcomatous differentiation was not described in this series. Binh et al reported 27 cases of DL with divergent myosarcomatous differentiation, including 7 cases in the paratesticular area, and leiomyosarcomatous differentiation was detected in 7 cases [9]. Henricks et al reported 155 cases of DL, including 13 cases of spermatic cord/ scrotum origin, and leiomyosarcomatous differentiation

Table 1 Clinicopathological features of well-documented cases of paratesticular liposarcoma with leiomyosarcomatous differentiation

\begin{tabular}{|c|c|c|c|c|c|c|c|c|c|}
\hline Case & Age (y) & Size $(\mathrm{cm})$ & $\begin{array}{l}\text { Classification } \\
\text { of liposarcoma }\end{array}$ & $\begin{array}{l}\text { Smooth muscle } \\
\text { component }\end{array}$ & Recurrences & Metastases & Status & Interval (mo) & Reference \\
\hline 1 & 49 & $7 \times 3.5 \times 3$ & ALTMDL & Well-differentiated & No & NA & NED & 30 & {$[6]$} \\
\hline 2 & 70 & $25 \times 21 \times 8$ & WDL & Moderately differentiated & $\mathrm{No}$ & No & NED & 72 & {$[7]$} \\
\hline 3 & 65 & 2 & WDL & Well-differentiated & Yes & No & NED & 96 & {$[8]$} \\
\hline Current case & 77 & $17.5 \times 12.5 \times 5$ & $\mathrm{DL}$ & Well-differentiated & No & No & NED & 24 & \\
\hline
\end{tabular}

$A L T$ atypical lipomatous tumor, WDL well-differentiated liposarcoma, $D L$ dedifferentiated liposarcoma, NED no evidence of disease. 
was observed in 2 of the 155 cases [11]. However, the relationship between the tumor location and the histological findings was not available in both studies.

To the best of our knowledge, only 3 well-documented cases of paratesticular liposarcoma with leiomyosarcomatous differentiation have been reported in the English literature [6-8]. The clinicopathological features of the reported cases, including the present case, are summarized in Table 1 . In case 1 , the tumor measured $7 \times 3.5 \times 3 \mathrm{~cm}$, and was composed of ALT/WDL with well-differentiated leiomyosarcomatous component. The smooth muscle cells were arranged in well-defined bundles with no prominent nuclear pleomorphism or mitosis. Immunostaining was not performed. In case 2, the tumor measured $25 \times 21 \times 8 \mathrm{~cm}$, and was composed of a WDL component with moderate leiomyosarcomatous differentiation. Proliferation of spindle cells, with enlarged, pleomorphic, and hyperchromatic nuclei were observed arranged in fascicles, with focal hemorrhage and necrosis in the leiomyosarcomatous area. On immunohistochemical examination, the leiomyosarcomatous components were positive for $\alpha \mathrm{SMA}$, desmin, and vimentin and negative for S-100 protein, keratin, CAM 5.2, and factor VIII-related antigen. In case 3, the tumor measured $2 \mathrm{~cm}$ and was composed of WDL component with leiomyosarcomatous differentiation. The smooth muscle component showed low cellularity and low nuclear grade. Immunohistochemical evaluation was not available. In the present case, the tumor was composed of a pleomorphic sarcomatous component with leiomyosarcomatous differentiation. Liposarcoma with smooth muscle differentiation was described relatively recently, and consists of 2 types: WDL having foci of mature but histologically atypical smooth muscle tissue (so-called lipoleiomyosarcoma) and DL with smooth muscle differentiation in the dedifferentiated areas (DL with heterogeneous differentiation) [8]. According to this classification, cases 1, 2, and 3 are lipoleiomyosarcoma, and the present case is DL with heterogeneous differentiation.

It is important to recognize the coexistence of the ALT/WDL components in order to obtain a definitive diagnosis of DL. However, if these components are not recognized, the differential diagnosis includes a variety of lesions, benign or low-grade mesenchymal tumors for low-grade differentiation, and pleomorphic sarcomas for high-grade differentiation. The general differential diagnosis of adult spindle cell tumors in this site includes primarily fibrous mesothelioma, leiomyosarcoma, malignant fibrous histiocytoma, various benign fibrous tumors, pseudotumors, and fibromatosis [2]. In addition to morphological findings, immunohistochemical study, including MDM2 and CDK4 staining, may be useful for diagnosis. The main karyotypic alteration of DL is considered to be the presence of supernumerary ring chromosomes and/or giant chromosomes composed of a chromosome 12q13-15 sequence involving MDM2 and often CDK4 genes [12]. The utility of MDM2 and CDK4 immunostaining is well documented in the diagnosis of WDL and DL [13]. However, MDM2 and CDK4 are not specific markers for liposarcoma. In the present case, the leiomyosarcomatous component showed no immunopositivity for MDM2 and CDK4; however, some leiomyosarcomas show positivity for MDM2 and CDK4 [3]. Therefore, it is important to prove the existence of the ALT/WDL component through additional sampling of the tumor.

Clinically, the risk of local recurrence is not affected by myosarcomatous differentiation in DL, although the metastatic rate is relatively low compared to that of leiomyosarcoma. The prognosis of patients with DL with myosarcomatous differentiation is better than that of patients with leiomyosarcoma [9]. Therefore, from a clinical perspective, it is important to distinguish DL with myosarcomatous differentiation from leiomyosarcoma in an argument for conventional adjuvant chemotherapy.

In summary, we report a rare case of paratesticular DL with with leiomyosarcomatous differentiation in a 77-year-old man. This case highlights the importance of additional sampling for obtaining a definitive diagnosis of DL, especially if the existence of an ALT/WDL component is not noted.

\section{Consent}

Written informed consent was obtained from the patient for publication of this case report and any accompanying images. A copy of the written consent is available for review by the Editor-in-Chief of this journal.

\section{Competing interests}

All authors declare that they have no competing interests.

\section{Authors' contributions}

$\mathrm{KH}$ and AT participated in conception of the idea and writing of the manuscript. TY and TT performed the histopathological interpretation of the tumor tissue. All authors read and approved the final manuscript.

\section{Acknowledgements}

We thank Dr. Masanori Hisaoka, Department of Pathology and Oncology, School of Medicine, University of Occupational and Environmental Health, for his thoughtful comments on the pathological diagnosis. This work was supported by a grant from the Kodama Memorial Fund for Medical Research.

Received: 25 July 2013 Accepted: 15 August 2013

Published: 23 August 2013

\section{References}

1. Brcić $L$, Jakovcević $A$, Vuletić $L B$, Orlić D, Seiwerth S: Pleomorphic liposarcoma of the foot: a case report. Diagn Pathol 2008, 3:15.

2. Montgomery E, Fisher C: Paratesticular liposarcoma: a clinicopathologic study. Am J Surg Pathol 2003, 27:40-47.

3. Dei Tos AP: Liposarcoma: new entities and evolving concepts. Ann Diagn Pathol 2000, 4:252-266.

4. Song JS, Gardner JM, Tarrant WP, Shen S, Ayala AG, Yu E, Ro JY: Dedifferentiated liposarcoma with peculiar meningothelial-like whorling and metaplastic bone formation. Ann Diagn Pathol 2009, 13:278-284. 
5. Li YF, Yu CP, Wu ST, Dai MS, Lee HS: Malignant mesenchymal tumor with leiomyosarcoma, rhabdomyosarcoma, chondrosarcoma, and osteosarcoma differentiation: case report and literature review. Diagn Pathol 2011, 6:35.

6. Evans HL: Smooth muscle in atypical lipomatous tumors. Am J Surg Pathol 1990, 14:714-718.

7. Suster S, Wong TY, Moran CA: Sarcomas with combined features of liposarcoma and leiomyosarcoma: study of two cases of an unusual soft-tissue tumor showing dual lineage differentiation. Am J Surg Pathol 1993, 17:905-911.

8. Flope AL, Weiss SW: Lipoleiomyosarcoma (well-differentiated liposarcoma with leiomyosarcomatous differentiation): a clinicopathologic study of nine cases including one with dedifferentiation. Am J Surg Pathol 2002, 26:742-749

9. Binh MB, Guillou L, Hostein I, Château MC, Collin F, Aurias A, Binh BN, Stoeckle E, Coindre JM: Dedifferentiated liposarcomas with divergent myosarcomatous differentiation developed in the internal trunk: a study of 27 cases and comparison to conventional dedifferentiated liposarcomas and leiomyosarcomas. Am J Surg Pathol 2007, 31:1557-1566.

10. Khoubehi B, Mishra V, Ali M, Motiwala H, Karim O: Adult paratesticular tumours. BJU Int 2002, 90:707-715.

11. Henricks WH, Chu YC, Goldblum JR, Weiss SW: Dedifferentiated liposarcoma: a clinicopathological analysis of 155 cases with a proposal for an expanded definition of dedifferentiation. Am J Surg Pathol 1997, 21:271-281

12. Pedeutour F, Forus A, Coindre JM, Berner JM, Nicolo G, Michiels JF, Terrier P, Ranchere-Vince D, Collin F, Myklebost O, Turc-Carel C: Structure of the supernumerary ring and giant rod chromosomes in adipose tissue tumors. Genes chromosomes. Cancer 1999, 24:30-41.

13. Binh MB, Sastre-Garau X, Guillou L, de Pinieux G, Terrier P, Lagacé R, Aurias A, Hostein I, Coindre JM: MDM2 and CDK4 immunostainings are useful adjuncts in diagnosing well-differentiated and dedifferentiated liposarcoma subtypes: a comparative analysis of 559 soft tissue neoplasms with genetic data. Am J Surg Pathol 2005, 29:1340-1347.

doi:10.1186/1746-1596-8-142

Cite this article as: Hatanaka et al.: Paratesticular dedifferentiated liposarcoma with leiomyosarcomatous differentiation: a case report with a review of literature. Diagnostic Pathology 2013 8:142.

\section{Submit your next manuscript to BioMed Central and take full advantage of:}

- Convenient online submission

- Thorough peer review

- No space constraints or color figure charges

- Immediate publication on acceptance

- Inclusion in PubMed, CAS, Scopus and Google Scholar

- Research which is freely available for redistribution 